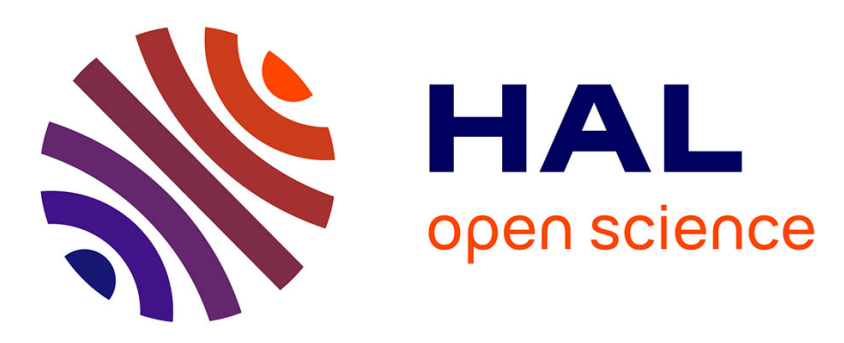

\title{
Modeling And Development Of A 4 Rotors Helicopter Uav
}

\author{
Naoufel Azouz, Khadidja Benzemrane, Gilney Damm, Gilbert Pradel
}

\section{To cite this version:}

Naoufel Azouz, Khadidja Benzemrane, Gilney Damm, Gilbert Pradel. Modeling And Development Of A 4 Rotors Helicopter Uav. IFAC Proceedings Volumes, 2007, 40 (15), pp.215-220. 10.3182/200709033-FR-2921.00039 . hal-03186134

\section{HAL Id: hal-03186134 https://hal.science/hal-03186134}

Submitted on 30 Mar 2021

HAL is a multi-disciplinary open access archive for the deposit and dissemination of scientific research documents, whether they are published or not. The documents may come from teaching and research institutions in France or abroad, or from public or private research centers.
L'archive ouverte pluridisciplinaire HAL, est destinée au dépôt et à la diffusion de documents scientifiques de niveau recherche, publiés ou non, émanant des établissements d'enseignement et de recherche français ou étrangers, des laboratoires publics ou privés. 


\title{
MODELING AND DEVELOPMENT OF A 4 ROTORS HELICOPTER UAV
}

\author{
Naoufel Azouz, Khadidja Benzemrane, \\ Gilney Damm and Gilbert Pradel
}

\author{
Laboratoire IBISC - CNRS/Université d'Evry Val \\ d'Essonne, 40 rue du Pelvoux 91020 Evry Cedex, France
}

\begin{abstract}
:
This paper presents the conception and construction of a mini 4 rotors helicopter for indoor and outdoor applications. The proposed UAV, named XSF, has a very maneuverable platform and is indicated to work in inaccessible spaces such as performing inspection tasks under bridges as well as inside pipes or tanks. Its main advantage with respect to classical 4 rotors helicopters is the ability of flipping two motors in order to obtain two more control inputs. This feature allows the XSF to have a better horizontal displacement or to create a yaw movement without translation. In this work we present the XSF's conception and implementation as well as its dynamic model, including the aerodynamic and gyroscopic effects.
\end{abstract}

Keywords: Unmanned aerial vehicles, robot modelling, embedded systems, robot automatic control.

\section{INTRODUCTION}

Recent development of small Unmanned Aerial Vehicles (UAV), have provided new possibilities for civil tasks such as search and rescue, survey and inspection. For the two last tasks, the rotor based aerial vehicles have an important advantage over conventional fixed wings aircrafts since they can easily hover above the target. In addition, in order to execute some specific tasks, the UAV should also be small and autonomous. With this goal, we discuss in this paper the design, modelling and control of a mini 4 rotors helicopter (quadrotor). This helicopter configuration is well known and there are a number of works dedicated to this subject as (Pounds et al., 2002) and (Castillo et al., 2004). The main reasons for using this configuration of UAV is its relatively mechanical simplicity in respect to the motors construction, with no need of complex mechanics in order to obtain a directional thrust, in particular for small drones. Furthermore, this con- figuration is highly symmetric, hence control and modeling are simplified. Finally, this UAV is much maneuverable and is mostly indicated to work in inaccessible spaces. The main applications for this configuration are then inspection under bridges and other buildings or structures, specially with non-smooth surfaces. Indoors supervision and inspection such as inside pipes or tanks are another important tasks for this kind of drones.

Most literature in the field of designing quadrotor helicopters is based on the development of indoor prototypes, see (Altug et al., 2002), (Castillo et al., 2004),(Guenard et al., 2005) and (Marti, 2000). However, the main problem associated with this concept is the instability of these prototypes face to atmospheric disturbances especially when maneuvering. In fact, quadrotors displacement is done in a quasi static attitude caused, in addition to physical reasons, by the difficulty of controlling such an aircraft. The main reasons of this difficulty are: the quite complex non-linear behavior 
of the quadrotor; the impossibility, in the general case, of measuring its internal state; the underactuated nature of the system. The first difficulty may be overcome by an improvement in the modeling phase. Actually, better understanding the system allow the development of improved robust and possible nonlinear controllers to ensure its stability. The second point will be discussed in section 3.1. Measurement or estimation of internal state is an important problem for small aerial vehicles, for which it is not possible to rely on the standard sensors as GPS. Finally, the third point is the underactuated nature of quadrotors which is the main motivation for the proposed structure. In fact, the addition of two control inputs is capital for the simplification of the control design step.

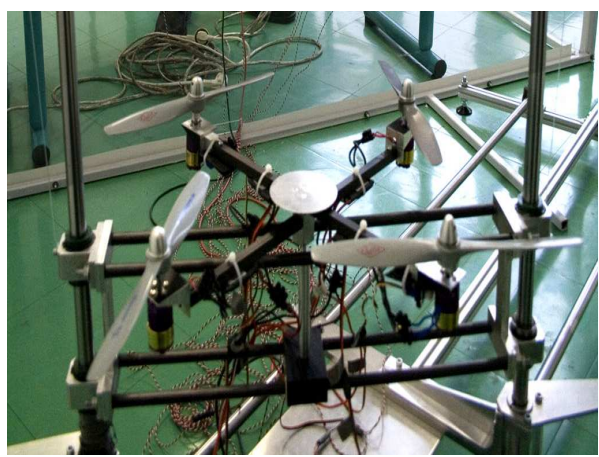

Fig. 1. The XSF and its test bed.

\section{DESIGN AND CONSTRUCTION}

The XSF drone (Figure 1)is a quadrotor with $0.68 \mathrm{~m} \times 0.68 \mathrm{~m}$. It is designed in the shape of a cross and was built in carbon fiber. Each end of the cross has a rotor including an electric brushless motor Flyware LRK-350/10, a motor speed controller Flyware rated $60 \mathrm{~A}$ at $12 \mathrm{~V}$ and a twoblade Graupner ducted propeller. Rotors (1) and (2) turn clockwise, while rotors (3) and (4) turn counterclockwise in order to maintain equilibrium and even be a control input in yaw motion. The main characteristic of the XSF compared to the existing quadrotors, is the swivelling of the supports of motors (1) and (4) around their pitching axis in a close manner of VTOL (Vertical TakeOff and Landing) planes. This allows an adequate horizontal flight and a supplementary control input for yaw motion.

In the center of the XSF, it is located a main cylinder enclosing the embedded sensors and microcontroler, namely an Inertial Measurement Unit, a GPS, ultrasound sensors and the $12 \mathrm{~V}$ LI-POLY battery cells. The embedded electronics is built around an ARM family processor running a realtime $e$-Cos operating system. In addition, a camera is used to provide image to a possible human pilot at a remote land-base. This pilot may give higher order tasks to the robot, waypoints or completely control it to better positioning of the camera in inspection tasks. The radio link connecting the embedded system to the remote base is made by a $2.4 \mathrm{GHz} \mathrm{RF}$ transmiter.

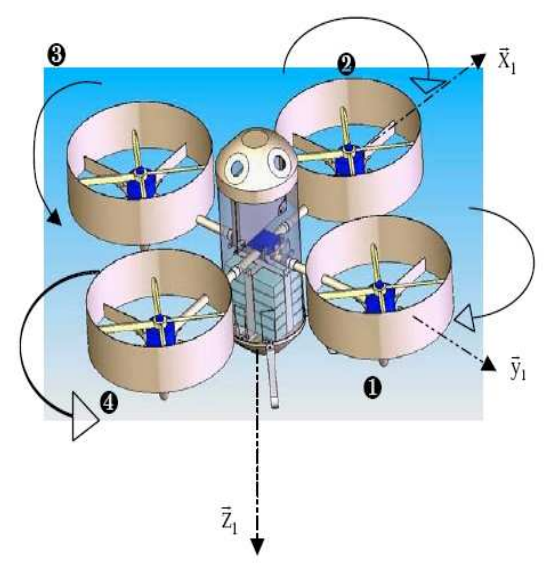

Fig. 2. Representation of the UAV

\subsection{Dynamic model}

The XSF drone is modelled first as a rigid flying object. We use here an eulerian approach, while a lagrangian approach can be seen in (Beji et al., 2006) and (Castillo et al., 2004):

We can first state:

$$
\begin{aligned}
\frac{d(m V)}{d t} & =\tau_{1} \\
\frac{d(\bar{I} \Omega)}{d t} & =\tau_{2}
\end{aligned}
$$

where $m$ represents the mass of the $\mathrm{UAV}, \bar{I}$ its inertia tensor, $V$ the linear speed in the center of gravity $G, \Omega$ the angular velocity of the local reference frame $(R L) \equiv(x, y, z)$ (figure 2 ) and $\tau_{1}$ $\tau_{2}$ the applied forces and moments, such as weight, aerodynamic forces and torques. Expressing the equation in the local reference frame obtains:

$$
\begin{aligned}
m\left(\frac{d(V)}{d t}+\Omega \wedge V\right) & =\tau_{1} \\
\bar{I} \frac{d(\Omega)}{d t}+\Omega \wedge \bar{I} \Omega & =\tau_{2}
\end{aligned}
$$




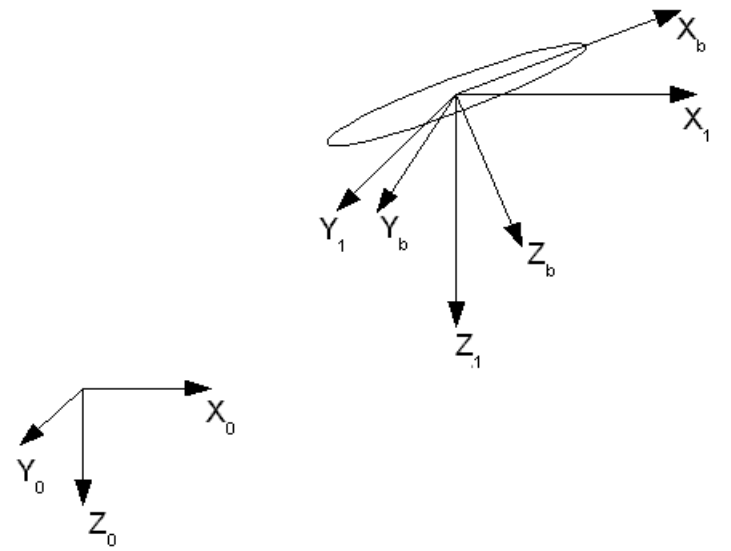

Fig. 3. Definition of the reference frames

Here we introduce three reference frames namely, the local reference frame $R_{b}=\left(x_{b}, y_{b}, z_{b}\right)$, an intermediate reference frame $R_{1}=\left(x_{1}, y_{1}, z_{1}\right)$, that is parallel to the inertial reference frame $R_{0}=\left(x_{0}, y_{0}, z_{0}\right)$. We have used the Euler angles $\psi, \theta$ and $\phi$ in order to express the relation betwen these different reference frames as:

$$
r=R^{T} r_{1}
$$

with

$R^{T}=\left(\begin{array}{ccc}c \theta . c \psi & c \theta . s \psi & -s \theta \\ s \phi . s \theta . c \psi-c \phi . s \psi & s \phi . s \theta . s \psi+c \phi . c \psi & s \phi . c \theta \\ c \phi . s \theta . c \psi+s \phi . s \psi & c \phi . s \theta . s \psi-s \phi . c \psi & c \phi . c \theta\end{array}\right)$

where $r$ is the position of a point in the local reference frame, and $r_{1}$ its position in the bodyfixed frame. Let's note $c \phi=\cos \phi$ and $s \phi=\sin \phi$.

The angular speed is given by:

$$
\Omega=\dot{\psi} \cdot e_{z_{1}}+\dot{\theta} \cdot e_{y_{2}}+\dot{\phi} \cdot e_{z_{3}}
$$

with $e_{y_{2}}$ an unitary vector resulting from the rotation $\psi$ of $\left(x_{1}, y_{1}, z_{1}\right)$ around $e_{z_{1}} ; e_{x_{3}}$ an unitary vector resulting from the rotation $\theta$ of $\left(x_{2}, y_{2}, z_{1}\right)$ around $e_{y_{2}}$. The angular speed may then be expressed as:

$$
\Omega=\left(\begin{array}{c}
p \\
q \\
r
\end{array}\right)=\left(\begin{array}{c}
\dot{\phi}-s \theta \cdot \dot{\psi} \\
s \phi \cdot c \theta \cdot \dot{\psi}+c \phi . \dot{\theta} \\
c \phi . c \theta \cdot \dot{\psi}-s \phi \cdot \dot{\theta}
\end{array}\right)
$$

In terms of the Euler angular velocities:

$$
\dot{\eta}=\left(\begin{array}{c}
\dot{\phi} \\
\dot{\theta} \\
\dot{\psi}
\end{array}\right)=\left(\begin{array}{ccc}
1 & s \phi . t g \theta & c \phi . t g \theta \\
0 & c \phi & -s \phi \\
0 & s \phi / c \theta & c \phi / c \theta
\end{array}\right)\left(\begin{array}{c}
p \\
q \\
r
\end{array}\right)
$$

We may then introduce $u, v$ and $w$ the linear speeds on the three axes of the local reference frame, such that $V=\left[\begin{array}{lll}u & v & w\end{array}\right]^{T}$, and then we obtain:

$$
\begin{aligned}
& m(\dot{u}+q w-r v)=X-m . g . s \theta \\
& m(\dot{v}+r u-p w)=Y+m . g . s \phi c \theta \\
& m(\dot{w}+p v-q u)=Z+m . g . c \phi c \theta
\end{aligned}
$$

with $X, Y$ and $Z$ the aerodynamic forces applied to the UAV.

We also introduce:

$$
\begin{aligned}
& I_{x x} \dot{p}+\left(I_{z z}-I_{y y}\right) q r+(\dot{r}+p q) I_{x z}+\left(q^{2}-r^{2}\right) I_{y z} \\
& +(\dot{q}-p r) I_{x y}=L \\
& I_{y y} \dot{q}+\left(I_{x x}-I_{z z}\right) r p+(\dot{p}+r q) I_{x y}+\left(r^{2}-p^{2}\right) I_{z x} \\
& +(\dot{r}-p q) I_{y z}=M \\
& I_{z z} \dot{r}+\left(I_{y y}-I_{x x}\right) p q+(\dot{q}+r p) I_{y z}+\left(p^{2}-q^{2}\right) I_{x y} \\
& +(\dot{p}-q r) I_{z x}=N
\end{aligned}
$$

$L, M$ and $N$ being the aerodynamic torques. Taking into account that the plan $x-z$ and $y-z$ are symmetry plans of the XSF, we may conclude that the inertia products $I_{x y}, I_{x z}$ and $I_{y z}$ are void. This fact allow us to simplify the previous system:

$$
\begin{aligned}
& I_{x x} \dot{p}=L-\left(I_{z z}-I_{y y}\right) q r \\
& I_{y y} \dot{q}=M-\left(I_{x x}-I_{z z}\right) r p \\
& I_{z z} \dot{r}=N-\left(I_{y y}-I_{x x}\right) p q
\end{aligned}
$$

The linear speed expressed in the fixed reference frame $\dot{r}=\left(\begin{array}{lll}\dot{x}_{0} & \dot{y}_{0} & \dot{z}_{0}\end{array}\right)^{T}$ may be expressed by the equation:

$\dot{x}_{0}=c \theta c \psi u+(s \phi s \theta c \psi-c \phi s \psi) v+(c \phi s \theta c \psi+s \phi s \psi) w$

$\dot{y}_{0}=c \theta s \psi u+(s \phi s \theta s \psi+c \phi c \psi) v+(c \phi s \theta s \psi-s \phi c \psi) w$

$\dot{z}_{0}=-s \theta u+s \phi c \theta v+c \phi c \theta w$

\subsection{Gyroscopic torques}

The gyroscopic effects of the rotating elements are introduced in the model as follows. Let's denote $\bar{I}_{R} \omega_{i}$ the kinetic moment of the rotor $(i)$, such as $\bar{I}_{R} \omega_{i} \triangleq\left[\begin{array}{lll}h_{x}^{i} & h_{y}^{i} & h_{z}^{i}\end{array}\right]^{T}$, then to incorporate these effects on the dynamic model it is rewritten as: 


$$
\begin{aligned}
& I_{x x} \dot{p}=L-\left(I_{z z}-I_{y y}\right) q r-q \sum_{i=1}^{4} h_{z}^{i}+r \sum_{i=1}^{4} h_{y}^{i} \\
& I_{y y} \dot{q}=M-\left(I_{x x}-I_{z z}\right) r p-r \sum_{i=1}^{4} h_{x}^{i}+p \sum_{i=1}^{4} h_{z}^{i} \\
& I_{z z} \dot{r}=N-\left(I_{y y}-I_{x x}\right) p q-p \sum_{i=1}^{4} h_{y}^{i}+q \sum_{i=1}^{4} h_{x}^{i}
\end{aligned}
$$

where we have used $I_{R}$ the inertia moment of the rotating elements in the rotors.

\subsection{Aerodynamic forces and torques}

In this section, we define the characteristics of the aerodynamic forces and torques issued from the blade theory.

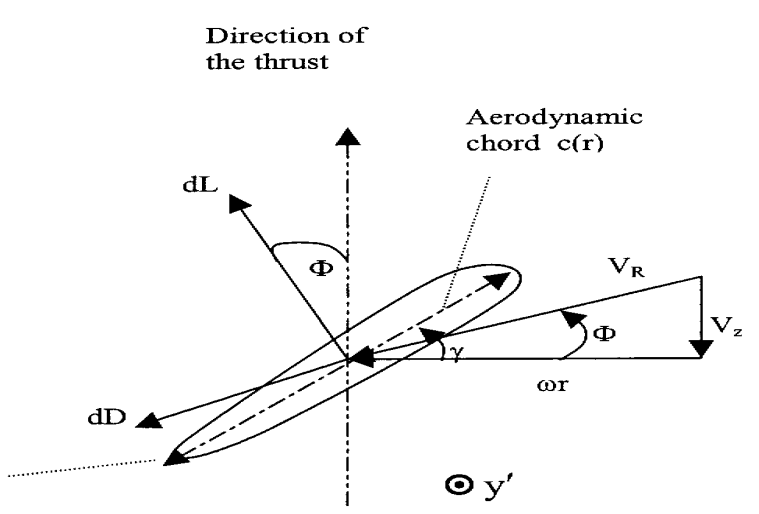

Fig. 4. Description of forces applied on the blade.

The blade behaves as a rotating wing. Each element of the blade $d r$ is in contact with the airflow. The local dynamic pressure at the blade element is : $q=\frac{1}{2} \rho(\omega r)^{2}$ and each elementary section of the blade of width $d r$ creates a lift $d L$ such as (see (Prouty, 1995)):

$$
d L=\frac{1}{2} \rho(\omega r)^{2} C_{L \alpha} c . \alpha . d r
$$

where $\rho$ is the air density, $C_{L \alpha}$ represents an adimensional coefficient of the gradient of lift, and the attack angle $\alpha$ is such that $\alpha=\gamma-\Phi$.

Furthermore, $\gamma$ is the geometric pitch of the blade, $\Phi$ the local inflow angle, and finally $c$ is the aerodynamic chord that were assumed constant here.

Since the inflow angle $\Phi$ is small, we have assumed that

$$
\tan \Phi=\frac{V_{z}}{\omega r} \Phi=\frac{V_{z}}{\omega R \frac{r}{R}}=\frac{\Phi_{t i p}}{\frac{r}{R}}
$$

We denote here $\Phi_{t i p}=\frac{V_{z}}{\omega R}$, with $R$ the length of the blade and $V_{z}$ the induced velocity. Similarly we suppose that the geometric pitch $\gamma$ varies related to its value in the tip of the blade such as:

$$
\gamma=\frac{\gamma_{t i p}}{r / R}
$$

Relation (11) becomes then:

$$
\begin{aligned}
d L & =\frac{1}{2} \rho(\omega r)^{2} C_{L \alpha} c . \frac{\gamma_{t i p}-\Phi_{t i p}}{r / R} . d r \\
& =\frac{1}{2} \rho \omega^{2} C_{L \alpha} c . \frac{\gamma_{t i p}-\Phi_{t i p}}{R} r . d r
\end{aligned}
$$

And integrating this last equation for the whole blade, one obtain:

$$
L_{\text {blade }}=\int_{0}^{R} d L=k_{1} \omega^{2}\left(\gamma_{t i p}-\Phi_{t i p}\right)
$$

With $k_{1}=\frac{1}{4} \rho R^{3} C_{L \alpha} c$. For a rotor with $B$ blades, the total thrust of the rotor is then given by:

$$
\bar{F}=B k_{1} \omega^{2}\left(\gamma_{t i p}-\Phi_{t i p}\right)
$$

In the other hand, during hovering, the induced velocity is given by (see (Padfield, 1996)): $V_{z}=$ $\sqrt{\frac{\bar{F}}{2 \rho A}}$ with $A$ the area of the rotor disc. This induces that:

$$
\Phi_{\text {tip }}=\frac{V_{z}}{\omega R}=\frac{k_{2} \bar{F}^{1 / 2}}{\omega}
$$

Here $k_{2}=\frac{1}{R \sqrt{2 \rho A}}$.

Equation (14) can be written as:

$$
\bar{F}=B k_{1} \omega^{2}\left(\gamma_{t i p}-\frac{k_{2} \bar{F}^{1 / 2}}{\omega}\right)
$$

that can be transformed in a second order equation in $\bar{F}^{1 / 2}$, resulting in:

$$
\bar{F}=\left(\frac{-k_{4}+\sqrt{\left(k_{4}\right)^{2} 04 k_{3}}}{2}\right)^{2} \cdot \omega^{2}
$$

with $k_{3}=B k_{1} \gamma_{t i p} \quad k_{4}=B k_{1} k_{2}$

Equation (17) can be re-defined simpler as:

$$
\bar{F} \triangleq k_{T} \omega^{2}
$$

The computation of the lift coefficient is often complex. Moreover marginal swirls at the tip of the blade can modify significantly the lift in this area. 
It was thus essential to elaborate an experimental process which would enable to determine the coefficient $\mathrm{kT}$ as well as the limits of validity of the relation (18).

Drag is defined similarly to lift, and produces a torque defined as

$$
M_{D}=k_{M} \omega^{2}
$$

The compensation of this torque in the center of gravity is done using the counter-rotating rotors 1-3 and 2-4. Recalling that by assumption we have $\omega_{3}, \omega_{4}<0$, these last two rotors having propellers with reversed pitch.

\subsection{Swivelling of rotors 1 and 3}

To allow a better horizontal displacement of the XSF without banking, we give the driving supports 1 and 3 an additional degree of freedom represented by their rotation around the $\boldsymbol{y}$ axis.

We denote by $\beta_{i}$ this swing angle. The two driving supports can either swivel in the same direction to create an horizontal component propelling the $\mathrm{XSF}$ in translation, or swivel in opposite directions to create a yaw movement without translation. The angles $\beta_{1}$ and $\beta_{3}$ are limited to 20 degrees, where this value was chosen because it maximazes energy in our application. The intensity lost on the vertical component of the force is about $6 \%$, while the horizontal component of the force represents $33 \%$. This component is big enough to thrust the XSF with the maximum desired speed $(10 \mathrm{~m} / \mathrm{s})$. Finally we should note that the swivelling of the driving supports has a negligible influence on the inertia terms. We assume then that the inertia terms are constant during the swivelling maneuver. .

\subsection{Complete Model}

We may now introduce the total dynamic model of the XSF taken into account the aerodynamic and gyroscopic effects, but not considering the effect of atmospheric wind $\left(u_{G}\right.$ and $l_{b}$ are physical lenghts):

$\dot{x}_{0}=c \theta c \psi u+(s \phi s \theta c \psi-c \phi s \psi) v+(c \phi s \theta c \psi+s \phi s \psi) w$

$\dot{y}_{0}=c \theta s \psi u+(s \phi s \theta s \psi+c \phi c \psi) v+(c \phi s \theta s \psi-s \phi c \psi) w$

$\dot{z}_{0}=-s \theta u+s \phi c \theta v+c \phi c \theta w$

$$
\begin{aligned}
& m \dot{u}=m(-q w+r v-g \cdot s \theta) \\
&-k_{T}\left(\omega_{1}^{2} \cdot \sin \beta_{1}+\omega_{3}^{2} \cdot \sin \beta_{3}\right) \\
& m \dot{v}=m(-r u+p w+g \cdot s \phi c \theta) \\
& m \dot{w}=m(-p v+q u+g \cdot c \phi c \theta) \\
&-k_{T}\left(\omega_{1}^{2} \cos \beta_{1}+\omega_{2}^{2}+\omega_{3}^{2} \cos \beta^{3}+\omega_{4}^{2}\right) \\
& \dot{\eta}=\left(\begin{array}{c}
\dot{\phi} \\
\dot{\theta} \\
\dot{\psi}
\end{array}\right)=\left(\begin{array}{ccc}
1 & s \phi \cdot t g \theta & c \phi \cdot t g \theta \\
0 & c \phi & -s \phi \\
0 & s \phi / c \theta & c \phi / c \theta
\end{array}\right)\left(\begin{array}{l}
p \\
q \\
r
\end{array}\right)
\end{aligned}
$$

and

$$
\begin{aligned}
I_{x x} \dot{p} & =-l_{b} k_{T}\left(\omega_{1}^{2} \cdot \cos \beta_{1}-\omega_{3}^{2} \cdot \cos \beta_{3}\right)-\left(I_{z z}-I_{y y}\right) r q \\
& -q I_{R}\left(\omega_{1} \cos \beta_{1}+\omega_{2}+\omega_{3} \cos \beta_{3}+\omega_{4}\right) \\
I_{y y} \dot{q} & =l_{b} k_{T}\left(\omega_{2}^{2}-\omega_{4}^{2}\right)-\left(I_{x x}-I_{z z}\right) r p \\
& -r I_{R}\left(\omega_{1} \sin \beta_{1}+\omega_{3} \sin \beta_{3}\right) \\
& +p I_{R}\left(\omega_{1} \cos \beta_{1}+\omega_{2}+\omega_{3} \cos \beta_{3}+\omega_{4}\right) \\
& +k_{M}\left(\omega_{3}^{2} \sin \beta_{3}-\omega_{1}^{2} \sin \beta_{1}\right) \\
& +u_{G} k_{T}\left(\omega_{1}^{2} \cdot \sin \beta_{1}+\omega_{3}^{2} \cdot \sin \beta_{3}\right) \\
I_{z z} \dot{r} & =-l_{b} k_{T}\left(\omega_{1}^{2} \cdot \sin \beta_{1}-\omega_{3}^{2} \cdot \sin \beta_{3}\right)-\left(I_{y y}-I_{x x}\right) p q \\
& +q I_{R}\left(\omega_{1} \sin \beta_{1}+\omega_{3} \sin \beta_{3}\right) \\
& +k_{M}\left(\omega_{3}^{2} \sin \beta_{3}+\omega_{4}^{2}-\omega_{1}^{2} \sin \beta_{1}-\omega_{2}^{2}\right)
\end{aligned}
$$

This model's parameters have been identified on our test bed as:

$$
\begin{array}{ll}
m=2.500 \mathrm{Kg} & k_{t}=1 e^{-5} N . s^{2} \\
l_{b}=0.23 m & I_{R}=100 e^{-7} \mathrm{Kgm}^{2} \\
I_{x x}=224931 e^{-7} \mathrm{Kgm}^{2} & I_{y y}=222611 e^{-7} \mathrm{Kgm}^{2} \\
I_{z z}=325130 e^{-7} \mathrm{Kgm}^{2} & k_{M}=9 e^{-5} \mathrm{Ns}^{2} \mathrm{~m} \\
u_{G}=0.032 \mathrm{~m} &
\end{array}
$$

where $l_{b}$ is the length of each arm of the drone while $u_{G}$ represents the distance between the center of gravity and the geometric center of the drone.

\section{CONTROL STRATEGY}

The foreseen applications for the XSF are based on a remote human operator that gives high level orders to the UAV, without needing to completely pilot it. A wireless link between a base-station and the UAV allows the operator to send navigation orders. Neverthless, the link can be cut if the UAV goes behind an obstacle and is hidden to the base. For this reason, the stabilizing controller must be on-board and not in the base-station. This stabilizing controller is based on classical robust control techniques. In the future, we intend to include pre-defined trajectories such that the drone may chose a behavior (fly higher, fly lower) to recover the link to the base. If this link is not recover after a pre-defined delay, an emergency procedure is chosen to drive the XSF to a soft 
descent to land. Another problem we are currently working on is optimazing the energy consumption as well as the measurement of the remaining energy in the battery cells. At any time this measurement is made to inform both the basestation and the on-board system. When a low level is reached, the on-board controller has to land the UAV.

\subsection{Observers}

Most control algorithms for quadrotors (see (Guenard et al., 2005), (Lozano et al., 2004)) need the drone's internal states. While the angles and angular velocities are readily available, position and linear velocities may be delicate to obtain. The standard solution is to use GPS sensors which may present a large error depending in the environment of the drone. These errors are usually small compared to the size of the vehicle and the distance to obstacles. Unfortunately, these techniques are not applyable for small drones, less than 1 meter wide, flying at low speed. This is even more relevant in indoors, or just urban applications. In these cases, the errors produced by a GPS system may reach many meters. This is a classical problem that was dealt with by the application of Kalman filters, which are not very effectible in the case of small drones. Recently, many works (see for instance (Zhao and Slotine, 2005) and (Vik and Fossen, 2001)) were consecrated to the development of observers in order to dynamically estimate at least part of the state vector. We have followed this direction and we have recently proposed an robust nonlinear adaptive observer (Benzemrane et al., 2007) that will be implemented on the embedded microprocessor, in order to be coupled in the robot's stabilization control algorithm. This observer was designed based on the techniques presented in (Benzemrane, 2006), (Marino et al., JUNE 2001) and (Marino and Tomei, Jan. 1998) and its first results even in the presence of noise are very interesting.

\section{CONCLUSION}

We have presented the XSF drone, a novel 4 rotors flying robot which presents a new design compared to the classical quadrotor version. This small drone is semi-autonomous and may perform different tasks of supervision and inspection following high level commands from a remote human operator. We have presented its physical model, its mechanical construction, and its control strategy. For the moment, the proposed drone has only flown on a test bed, and future steps will be flying tests on outdoors, the coupling of the observer algorithm to the controller, and the human-machine interface in order to close the loop.

\section{REFERENCES}

Altug, E., J. Ostrowski and R. Mahony (2002). Control of a quadrotor helicopter using visual feedback. In: Proceedings of the IEEE Int. Conference on Robotics and Automation. ICRA 02. Washington DC, USA.

Beji, L., A. Abichou and N. Azouz (2006). Modelling, motion planning and control of the drones with revolving airfoils: an outline of the xsf. Robot Motion and Control Recent Developments Series: Lecture Notes in Control and Information Sciences.

Benzemrane, K. (2006). Systeme observateur pour un drone helicoptere. Master's thesis. Laboratoire IBISC - CNRS / Universite d'Evry Val d'Essonne. France. (In french).

Benzemrane, K., G.L. Santosuosso and G.Damm (2007). Unmanned aerial vehicle speed estimation via nonlinear adaptive observers. In: IEEE American Control Conference. NY.

Castillo, P., A. Dzul and R. Lozano (2004). Realtime stabilization and tracking of a four rotor mini-rotorcraft. IEEE Transactions on Control Systems Technology.

Guenard, N., T. Hamel and V. Moreau (2005). Dynamic modelling and intuitive control strategy for an x4-flyer. ICCA 05. Budapest.

Lozano, R., A. Dzul and P. Castillo (2004). Global stabilization of the pvtol : Real-time application to a mini-aircraft. 77, n. 8, 276-279.

Marino, R. and P. Tomei (Jan. 1998). Robust adaptive state-feedback tracking for nonlinear systems. IEEE Trans. Automatic Control 43, no. 1, 84-89.

Marino, R., G.L. Santosuosso and P. Tomei (JUNE 2001). Robust adaptive observers for nonlinear systems with bounded disturbances. IEEE Transactions on Automatic Control 46(6), 967-972.

Marti, S. (2000). The zero g eye: Towards a free hovering camera. In: Technical Report. MIT. Massachusetts, USA.

Padfield, G. D. (1996). Helicopter Flight Dynamics. Blackwell Science.

Pounds, P., R. Mahony, P. Hynes and J. Roberts (2002). Design of a four-rotor aerial robot. In: Procedings of 2002 Australasian Conference on Robotics and Automation. Auckland.

Prouty, R. W. (1995). Helicopter Performance, Stability and Control. Krieger Publishing Company.. USA.

Vik, B. and T.I. Fossen (2001). A nonlinear observer for gps and ins integration. In: Proceedings of the 40th IEEE Conference on Decision an Control. IEEE. Orlando, Florida, USA. pp. 2956-2961.

Zhao, Y. and J.J. Slotine (2005). Discrete nonlinear observers for inertial navigation. Systems and Control Letters. 